\title{
Pay Transparency: A Comparison of Employee Perceptions in Poland and the United States
}

\author{
Dow Scott ${ }^{1}$ Jacek Grodzicki ${ }^{2}$ \\ Loyola University Chicago ${ }^{1}$ \\ University of Gdansk ${ }^{2}$
}

\begin{abstract}
Although thought leaders have long advocated pay transparency, employers remain reluctant to share pay information for a variety of reasons, including concerns about employee privacy and exposure of pay structure inequity and shortcomings.(e.g., Colella et al. 2007; Day 2007, 2012; Futrell and Jenkins 1978;Scott and McMullen 2013).Pay transparency increasingly has become an expectation, especially among younger employees, because of increased access to pay information from websites like salary.com and monster.com, social media and legislation supporting employee access to this information (e.g., Scott, 2018). Furthermore, one might expect perception about pay transparency to differ across countries with different cultures, employment practices and legislative mandates. Numerous studies have examined pay transparency, secrecy and communications from the perspective of reward leaders (e.g., Case 2001; Futrell and Jenkins 1978; Gherson, 2000; Lawler, 1966; 1967; Sim 2001; Bierman and Gely 2004). However, few studies examine how employee feel about pay transparency and how those perceptions differ across countries (e.g., Hundley \& Kim, 1997; Tang, Luna-Arocas and Sutarso, 2005). Finally, much of this research is old and perceptions about pay transparency and secrecy may well have changed (Scott, 2018). Based upon a sample of full-time employees from two-countries (Poland, and U.S.), these perceptions are compared and important differences are identified. Initial findings indicate that pay communications and perceived pay transparency are related to trust in management, pay fairness, and pay satisfaction. How these perceptions differ across country is more complex. A complete reference list can be obtain from the authors.

Keywords: Pay transparency, fairness, satisfaction, secrecy, trust, communication.
\end{abstract}

